Authors: Dr Andrew Fulton, Dr Maya Amlani, Dr Susan Parekh

\title{
Oral manifestations of vitamin $D$ deficiency in children
}

In this paper, we highlight the importance of Vitamin D to the development of the permanent dentition,and discuss management options of cases with chronological enamel hypoplasia due to nutritional vitamin D deficiency.

\section{Vitamin D - An overview}

Vitamin D, along with Parathyroid hormone (PTH), fibroblast growth hormone 23 (FGF23), and calcitonin, are regulators of the calcium and phosphate metabolic pathways, which are key to the development and maintenance of the musculoskeletal system. ${ }^{1}$ There are two sources of vitamin D: via synthesis in the skin on exposure

to ultra-violet $B$ (UVB) radiation-containing-sunlight (primary source), and through nutritional sources. However, when there is insufficient exposure to sunlight or the levels of UVB are low, such as during winter, then dietary sources become more important. ${ }^{2}$ It is not possible to synthesis vitamin $D$ when exposed to sunlight through a window as glass absorbs UVB. ${ }^{3}$

Vitamin D sufficiency is generally regarded as being serum $>50 \mathrm{nmol} / \mathrm{L}$, and deficiency as $<25 \mathrm{nmol} / \mathrm{L}$. 4,5 The systemic impacts of vitamin $D$ deficiency are numerous, though the most common in children is the bone deformity rickets, and in adults, osteomalacia.

It is important to note that hypophosphatemic rickets (vitamin D resistant rickets) differs from vitamin $D$ deficiency rickets. In hypophosphatemic rickets, production of vitamin $D$ is inhibited secondary to phosphate wasting, which is caused by mutations in a range of genes (mainly PHEX) and is commonly inherited. Treatment is with vitamin D analogs and phosphate supplements. ${ }^{6}$

A range of risk factors are associated with nutritional vitamin D deficiency including; female gender, age, premature birth, skin pigmentation, obesity, malabsorption and advanced age, living in large cities and rural areas. ${ }^{7}$ Whilst low sunlight exposure is a risk factor for everyone living in the UK especially during winter, people with a darker skin tone need to spend more time in the sun relative to those with lighter skin in order to synthesis the same amount of vitamin D. ${ }^{3}$

Recent studies have suggested a genetic link to vitamin D deficiency, with data from twin and family-based studies reporting that circulating vitamin D concentrations are partially determined by genetic factors. However, the underlying genetic mechanism related with to serum $25(\mathrm{OH}) \mathrm{D}$ concentrations remain poorly understood, and further research is needed. ${ }^{8}$

Maternal vitamin D status is a factor both prenatally and for neo-nates, with maternal vitamin $D$ deficiency associated with increased risk of pre-eclampsia, gestational 
diabetes mellitus, postpartum depression, emergency caesarean section delivery, low birth weight, and neonatal hypoglycaemia. ${ }^{9,10}$

Rates of nutritional vitamin $D$ related rickets have increased from 0.34 cases per 100,000 children between 1991 and 1996 to 3.16 cases per 100,000 between 2007 and 2011 in the UK. ${ }^{11}$ This is not an issue solely confined to the UK, a pan-European study found that up to $40.4 \%$ of the population are vitamin $D$ deficient, with people from dark-skinned ethnic sub-groups being a significantly higher proportion of people affected. ${ }^{12}$

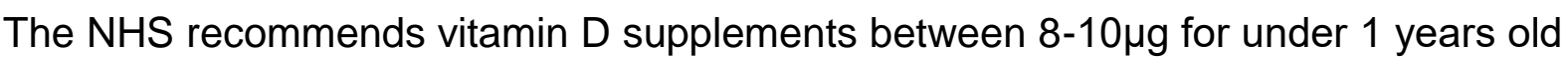
(unless the infant is consuming infant formula, as it is fortified with vitamin D), and $10 \mu \mathrm{g}$ for those aged 1-4 years. In addition, those that do not often go outside, or frequently cover up their skin with clothing, should also receive such supplementation. The rest of the population is advised to consider taking supplements, especially during the Winter months. ${ }^{3}$

\section{Vitamin D deficiency and Oral Health}

\section{Enamel Defects}

Patients presenting with childhood vitamin $\mathrm{D}$ deficiency rickets are more likely to have enamel defects compared to controls. ${ }^{13}$ Low vitamin $D$ levels are a risk factor for hypoplasia (a quantity defect resulting in missing / pitted enamel) in the permanent dentition. ${ }^{14}$ Another study of mothers with lower vitamin $D$ levels during pregnancy, suggested a possible link with enamel hypoplasia affecting the primary maxillary central incisors. ${ }^{15}$

Hypoplasia caused by vitamin D deficiency has the distinct characteristic presentation of "banding", termed chronological hypoplasia. The hypoplastic enamel appears as bands across the crowns of the teeth, and corresponds to the section of enamel that was being produced during the time of the deficiency. When the deficiency is corrected, enamel production returns to normal, and so a clear distinction exists between these two sections, as demonstrated in figure 1-A and 1-B. Radiographs show the characteristic enamel banding of affected teeth, and normal enamel of teeth developing after the vitamin D deficiency is corrected. Figure 2 shows a DPT of a 9 year girl diagnosed at 15 months of age, with the first permanent molars, permanent incisors and canine tips affected, but premolars and second permanent molars unaffected.

We can use the position of the bands on affected teeth to determine at which age the patient may have suffered from vitamin $D$ deficiency. Often the position of the bands will vary on different teeth (although will almost always have a symmetrical distribution), correlating with the timing of tooth development, with the first permanent molars, incisors and canines the first adult teeth to develop after birth. ${ }^{16} \mathrm{~A}$ common 
presentation is for the incisal half of the incisor teeth, tips of the permanent canines and the occlusal half of the first permanent molar teeth to be affected.

It is important to note that hypoplastic teeth can have various aetiologies, and so the diagnosis of chronological hypoplasia associated with a vitamin $\mathrm{D}$ deficiency is only given to those with a diagnosis of vitamin D deficiency, or a history of delayed walking and bowing of the legs when infants.

There is conflicting data regarding the association between vitamin $\mathrm{D}$ deficiency and Molar-Incisor Hypomineralisation (MIH). One study found that increased levels of vitamin Dcorrelated with a decreased incidence of $\mathrm{MIH},{ }^{17}$ while another looking at foetal, neonatal and childhood vitamin D levels, found no correlation with $\mathrm{MIH} .{ }^{18}$

\section{Caries}

A study looking at low maternal serum vitamin $D$ levels during pregnancy found a significant association $(P=0.05)$ to early childhood caries among infants up to 1 years old. ${ }^{19}$ Low vitamin $D$ levels have also been shown to be associated with high caries risk, and the severity of decay in primary/mixed dentitions, ${ }^{20,21,22}$ though the evidence for the permanent dentition is limited and further investigation is required. ${ }^{23}$

\section{Periodontitis}

In adults, vitamin D sufficiency can decrease the risk of gingivitis and chronic periodontitis, as it has immunomodulatory, anti-inflammatory, antiproliferative effects and initiates cell apoptosis. ${ }^{24}$ While there is evidence to support the importance of vitamin $\mathrm{D}$ to periodontal health in adults, ${ }^{25}$ there is none for children at present.

\section{Orthodontics}

There is currently no evidence to suggest that vitamin D levels has any significant correlation with external-induced root resorption in patients undergoing orthodontic treatment. ${ }^{26}$

\section{Management of children with enamel defects due to vitamin D deficiency}

\section{Minimal \& No Preparation Treatment Techniques}

Common complaints from patients with enamel defects include, aesthetic concerns due to unusually shaped teeth, as well as sensitivity arising from exposed dentine. Treatment should focus on early diagnosis and preventive care. Children with chronological hypoplasia require more frequent dental check-ups to reduce the risk of dental decay. ${ }^{27}$

Fluoride varnish applications, sealants and use of remineralizing agents, for example casein phosphopeptide amorphous calcium phosphate (CPP-ACP), may help to reduce the risk of dental caries and sensitivity. ${ }^{28,29}$ 
If treatment is required, options include adhesive restorations for anterior and minimally affected posterior teeth, (as the remaining enamel is mineralised, and therefore bonding is possible). Tooth preparation is not usually required, as the missing enamel and banding, give the appearance of a 'prepped tooth' as in Figures $1 \mathrm{~A} \& 1 \mathrm{C}$. Primary and permanent molar teeth that are more extensively affected can require stabilisation with preformed metal crowns, which can be placed using minimally invasive/Hall techniques. Teeth with extensive enamel hypoplasia in the permanent dentition, particularly first permanent molars (FPMs) of poor prognosis, may require extraction. In such cases, interdisciplinary team management involving specialist paediatric dentists and orthodontists may be necessary. ${ }^{30}$ The use of vital bleaching, and/or icon infiltration treatments are generally unsuitable for patients presenting with chronological hypoplasia, as the primary concern is missing enamel, and so these interventions will have limited/no impact. Microabrasion may be useful in removing surface staining in hypoplasia cases,$^{31}$ though restoration will still likely be needed after. ${ }^{32}$

\section{Case Examples - Anterior teeth}

In figure 1-C, the 7 year old female patient was complaining of "brown teeth" and generalised sensitivity for the preceding 6 months when drinking cold drinks. Her medical history included a previous diagnosis of rickets, due to difficulties walking, which was treated at 14 months of age with vitamin $D$ supplements. The patient was otherwise fit and well, and no longer taking vitamin D supplements. There is no familial history of rickets or enamel hypoplasia.

Clinical examination showed an early mixed dentition with suboptimal hygiene leading to generalised plaque induced gingivitis. Figure 1-C shows hypoplasia effecting the maxillary and mandibular central incisors and the lower laterals. The patient was of Afro-Caribbean origin and there is evidence of generalised physiological gingival pigmentation present. The hypoplasia appears as a chronological band, with sound enamel present in the cervical thirds.

Sensitivity reported by the patient was determined to relate to the upper central incisors, and these were the teeth that most bothered the patient in terms of appearance. It was decided to postpone treatment of the lower central incisors until the lateral incisors had erupted, as these teeth were not causing the patient any discomfort. The upper central incisors were restored with direct composite (shade A1) using crown forms under local anaesthetic (figure 1-D). Fluoride varnish was placed on the lower incisors.

Where the Vitamin $D$ deficiency has lasted longer, the entire enamel layer can be affected by hypoplasia, as can be seen in Figure 1-E. This 10 year old male patient was unhappy with the appearance of his anterior teeth as they were 'small and yellow'. He had been diagnosed with vitamin D deficiency at the age of 2-3 due to 'issues with his legs', with vitamin $D$ supplements subsequently prescribed for 3 years. 
Hypoplasia of the whole enamel layer can be seen in the upper central incisors, while the upper lateral and lower incisors have a banded appearance (figure 1-F), indicative of the chronological nature of Vit $\mathrm{D}$ deficiency. This banding in the lower incisors means Amelogenesis Imperfecta is excluded from the differential diagnosis. The tips of the partially erupted canines are also hypoplastic.

In this case, all the incisors were built up with direct composite, with the canines planned for after further eruption (figures $1-\mathrm{G} \& 1-\mathrm{H}$ ).

\section{Case Examples - Posterior teeth}

In figures 1-land1-K (patient 2), the upper and lower right first permanent molars show hypoplasia of the whole coronal surface and caries interproximally with cavitation. The upper and lower left FPMs were less severely affected by hypoplasia and had no caries, with a class 1 incisal relationship.

The maxillary and mandibular right first permanent molars were extracted while the left molars were preserved, and $\mathrm{OHI} /$ fluoride varnish administered.

In figures $1-\mathrm{F}$ and $1-\mathrm{J}$, a 10 year old male patient presented with pain in the lower right quadrant, which had been ongoing for 1-2 months.

All four first permanent molars are hypoplastic, with a thin band of sound enamel present at the cervical margins, and a class 1 incisal relationship.

As the lower second permanent molars had erupted, following orthodontic assessment, it was decided to restore the first permanent molars rather than extract them as mesialisation would not be likely to occur. Due to the extent of hypoplasia and presence of caries in some of the molars, it was decided to crown these teeth using the Hall technique in order preserve as much tooth structure as possible. This required the use of separators, and the crimping of the crowns to ensure a tight marginal seal (figure 1-H).

\section{Conclusion}

Vitamin $D$ deficiency in infancy is increasing,and can affect the appearance and prognosis of the permanent teeth. Enamel defects resulting from vitamin $D$ deficiency can appear as chronological banding or affect the whole tooth.

Treatment options for anterior teeth involve composite restorations to restore shape and aesthetics, while options for molars include composite restorations, preformed metal crowns, and extractions. If extraction of poor prognosis teeth is required, then a multidisciplinary approach is essential in the treatment planning stage. 
Dentists and medical teams need to be aware of the implications of vitamin D deficiency in early childhood, and supplements should be considered for high risk groups.

\section{Bibliography}

1) Girgis $C$, Clifton-Bligh R, Hamrick M, Holick M, Gunton J. The Roles of Vitamin D in Skeletal Muscle: Form, Function, and Metabolism. Endocrine Reviews. 2013;34(1):33-83.

2) Vitamin D and Health Report [Internet]. Scientific Advisory Committee on Nutrition. 2019 [cited 3 September 2019]. Available from:

https://assets.publishing.service.gov.uk/government/uploads/system/uploads/attachment dat a/file/537616/SACN Vitamin D and Health report.pdf

3) How to get vitamin D from sunlight [Internet]. nhs.uk. 2018 [cited 3 September 2019]. Available from: https://www.nhs.uk/live-well/healthy-body/how-to-get-vitamin-d-from-sunlight/

4) Munns C, Shaw N, Kiely M, Specker B, Thacher T, Ozono K et al. Global Consensus Recommendations on Prevention and Management of Nutritional Rickets. The Journal of Clinical Endocrinology \& Metabolism. 2016;101(2):394-415.

5) Braegger C, Campoy C, Colomb V, Decsi T, Domellof M, Fewtrell M et al. Vitamin D in the Healthy European Paediatric Population. Journal of Pediatric Gastroenterology and Nutrition. 2013;56(6):692-701.

6) Linglart A, Biosse-Duplan M, Briot K, Chaussain C, Esterle L, Guillaume-Czitrom S et al. Therapeutic management of hypophosphatemic rickets from infancy to adulthood. Endocrine Connections. 2014;3(1):R13-R30.

7) Lips P. Vitamin D physiology. Progress in Biophysics \& Molecular Biology. 2006;92(1):4-8

8) Bahrami A, Sadeghnia H, Tabatabaeizadeh S, Bahrami-Taghanaki H, Behboodi N, Esmaeili $\mathrm{H}$ et al. Genetic and epigenetic factors influencing vitamin D status. Journal of Cellular Physiology. 2017;233(5):4033-4043.

9) van der Pligt P, Willcox J, Szymlek-Gay E, Murray E, Worsley A, Daly R. Associations of Maternal Vitamin D Deficiency with Pregnancy and Neonatal Complications in Developing Countries: A Systematic Review. Nutrients. 2018;10(5):640.

10) Dovnik A, Mujezinović $F$. The Association of Vitamin D Levels with Common Pregnancy Complications. Nutrients. 2018;10(7):867.

11) Goldacre M, Hall N, Yeates D. Hospitalisation for children with rickets in England: a historical perspective. The Lancet. 2014;383(9917):597-598.

12) Cashman K, Dowling K, Škrabáková Z, Gonzalez-Gross M, Valtueña J, De Henauw $S$ et al. Vitamin D deficiency in Europe: pandemic?. American Journal of Clinical Nutrition. 2019;104(4):1033-1044.

13) Zerofsky M, Ryder M, Bhatia S, Stephensen C, King J, Fung E. Effects of early vitamin D deficiency rickets on bone and dental health, growth and immunity. Maternal \& Child Nutrition. 2015;12(4):898-907.

14) Ford $D$, Seow $W$, Kazoullis $S$, Holcombe $T$, Newman B. A controlled study of risk factors for enamel hypoplasia in the permanent dentition. Paediatric Dentistry. 2009;31(1):382-388.

15) Reed S, Voronca D, Wingate J, Murali M, Lawson A, Hulsey T et al. Prenatal vitamin D and enamel hypoplasia in human primary maxillary central incisors: A pilot study. Pediatric Dental Journal. 2017;27(1):21-28.

16) Watts $A$, Addy M. Tooth discolouration and staining: a review of the literature. British Dental Journal. 2001;190(6):309-316.

17) Kühnisch J, Thiering E, Kratzsch J, Heinrich-Weltzien R, Hickel R, Heinrich J. Elevated serum $25(\mathrm{OH})$-vitamin $\mathrm{D}$ levels are negatively correlated with molar-incisor hypomineralization. Journal of Dental Research. 2015;94(2):381-387. 
18) van der Tas J, Elfrink M, Heijboer A, Rivadeneira F, Jaddoe V, Tiemeier $\mathrm{H}$ et al. Foetal, neonatal and child vitamin $\mathrm{D}$ status and enamel hypomineralization. Community Dentistry and Oral Epidemiology. 2018;46(4):343-351.

19) Schroth $R$, Lavelle $C$, Tate $R$, Bruce $S$, Billings $R$, Moffatt M. Prenatal Vitamin $D$ and Dental Caries in Infants. Pediatrics. 2014;133(5):1277-1284.

20) Schroth R, Levi J, Sellers E, Friel J, Kliewer E, Moffatt M. Vitamin D status of children with severe early childhood caries: a case-control study. BMC Pediatrics. 2013;13(1).

21) Schroth R, Rabbani R, Loewen G, Moffatt M. Vitamin D and Dental Caries in Children. Journal of Dental Research. 2015;95(2):173-179.

22) Gupta A, Chhonkar A, Arya V. Comparison of Vitamin D Level of Children with Severe Early Childhood Caries and Children with No Caries. International Journal of Clinical Pediatric Dentistry. 2018;11(3):199-204.

23) Kim I, Lee H, Ju H, Na J, Oh H. A cross-sectional study on the association between vitamin $D$ levels and caries in the permanent dentition of Korean children. BMC Oral Health. 2018;18(1).

24) Alshouibi E, Kaye E, Cabral H, Leone C, Garcia R. Vitamin D and Periodontal Health in Older Men. Journal of Dental Research. 2013;92(8):689-693.

25) Jagelavičienė E, Vaitkevičienè I, Šilingaitè D, Šinkūnaitè E, Daugèlaitė G. The Relationship between Vitamin D and Periodontal Pathology. Medicina. 2018;54(3):45.

26) Younessian F, Tehranchi A, Sadighnia A, Abdi A, Shirvani A. Correlation of vitamin D status and orthodontic-induced external apical root resorption. Dental Research Journal. 2017;14(6):403.

27) Foulds $\mathrm{H}$. Developmental defects of enamel and caries in primary teeth. Evidence-Based Dentistry. 2017;18(3):72-73.

28) Adair S. Evidence-based use of fluoride in contemporary pediatric dental practice. Paediatric Dentistry. 2006;28(1):133-142.

29) Tao S, Zhu Y, Yuan H, Tao S, Cheng Y, Li J et al. Efficacy of fluorides and CPP-ACP vs fluorides monotherapy on early caries lesions: A systematic review and meta-analysis. PLOS ONE. 2018;13(4):e0196660.

30) Salanitri S, Seow W. Developmental enamel defects in the primary dentition: aetiology and clinical management. Australian Dental Journal. 2013;58(2):133-140.

31) Reston E, Corba D, Ruschel K, Tovo M, Barbosa A. Conservative Approach for Esthetic Treatment of Enamel Hypoplasia. Operative Dentistry. 2011;36(3):340-343.

32) Pini N. Enamel microabrasion: An overview of clinical and scientific considerations. World Journal of Clinical Cases. 2015;3(1):34. 
